\title{
On Finite Representations of Infinite-State Behaviours
}

\author{
Antonín Kučera ${ }^{\star}$ \\ e-mail: tony@fi.muni.cz \\ Faculty of Informatics, Masaryk University \\ Botanická 68a, 60200 Brno \\ Czech Republic
}

\begin{abstract}
We examine the problem of finite-state representability of infinitestate processes w.r.t. certain behavioural equivalences. We show that the classical notion of regularity becomes insufficient in case of all equivalences of van Glabbeek's hierarchy except bisimilarity, and we design and justify a generalization in the form of strong regularity and finite characterizations. We show that the condition of strong regularity guarantees an existence of finite characterization in case of all equivalences of van Glabbeek's hierarchy, and we also demonstrate that there are behaviours which are regular but not strongly regular w.r.t. all equivalences of the mentioned hierarchy except bisimilarity.
\end{abstract}

\section{Introduction}

The problem whether a given infinite-state behaviour (process) can be equivalently represented by a finite-state one has recently attracted a lot of attention. A similar problem has been actually known from the theory of formal languages for a long time-given a grammar $G$, one can ask whether there is an equivalent regular grammar $G^{\prime}$. The grammar $G^{\prime}$ can be seen as a 'finite-state representation' of $G$ because of the associated finite-state automaton. However, it is folklore that the mentioned problem is undecidable even for context-free grammars.

The situation is more complicated within the framework of concurrency theory. Transition systems are widely accepted as structures which can exactly define semantics of concurrent process; however, there are many behavioural equivalences over the class of transition systems which try to formally express 'sameness' of two concurrent systems. Rob van Glabbeek presented in [vG90] a hierarchy of equivalences, relating them w.r.t. their coarseness (see Figure 1).

The problem whether for a given process there is an equivalent finite-state one has been intensively studied w.r.t. bisimulation equivalence (bisimilarity); it is also known as the "regularity problem". Regularity has been proved to be decidable for BPA processes [MM94,BG96,BCS96], labelled Petri nets (and thus also BPP processes) [JE96], normed PA processes [Kuč96], and one-counter processes [Jan97]. Those results are also interesting from the practical point of view-verification of infinite-state systems is generally difficult, but if we replace an infinite-state system with some equivalent finite-state one, the procedure can be much easier. Moreover, decidability

\footnotetext{
* Supported by GA ČR, grant number 201/97/0456
} 
of regularity can simplify various considerations about infinite-state behaviours (see e.g., [ČKK 97,Kuč97]).

In this paper we examine a general question what properties should have a finitestate transition system if it is to be used as a 'reliable' description of some infite-state one. We argue that in case of all equivalences of van Glabbeek's hierarchy except bisimilarity the notion of regularity becomes insufficient, as it does not characterize reachable states (see the first paragraph of Section 3). We design and justify a new notion of finite characterization and we examine its basic properties. We prove that the condition of strong regularity guarantees an existence of a finite characterization w.r.t. all equivalences of van Glabbeek's hierarchy. As regularity and strong regularity coincide in case of bisimilarity, the condition of strong regularity can be seen as a 'proper' predicate expressing the feature of finite representability. We also prove that regularity and strong regularity do not coincide in case of all equivalences of van Glabbeek's hierarchy except bisimilarity, i.e., strong regularity is really a 'stronger' condition than regularity. We conclude with some remarks on future work.

\section{Definitions}

Definition 1. A transition system $T$ is a tuple $(S, A c t, \rightarrow, r)$ where $S$ is a set of states, Act is a set of labels, $\rightarrow \subseteq S \times A c t \times S$ is a transition relation and $r \in S$ is a distinguished state called root. The class of all transition systems is denoted by $\mathcal{T}$.

As usual, we write $s \stackrel{a}{\rightarrow} t$ instead of $(s, a, t) \in \rightarrow$ and we extend this notation to elements of $A c t^{*}$ in an obvious way (we sometimes write $s \rightarrow^{*} t$ instead of $s \stackrel{w}{\rightarrow} t$ if $w \in A c t^{*}$ is irrelevant). A state $t$ is said to be reachable from a state $s$ if $s \rightarrow^{*} t$. The states which are reachable from the root are said to be reachable.

Various behavioural equivalences over the class of transition systems were proposed in the literature - each of them tries to express a certain level of 'sameness' which is proper in certain situations. Rob van Glabbeek presented in [vG90] a hierarchy of behavioural equivalences, relating them w.r.t. their coarseness, i.e., how many identifications they make. The resulting lattice is presented in Figure 1.

Definition 2. Let $T$ be a transition system and let $\leftrightarrow$ be an equivalence over $\mathcal{T}$. The system $T$ is regular w.r.t. $\leftrightarrow$ if there is a finite-state transition system $F$ such that $T \leftrightarrow$ $F$. Such a system $F$ is called a finite representation of $T$.

\section{Finite Characterizations}

The notion of finite representation can be used for any equivalence of van Glabbeek's hierarchy. It is extremely useful in case of bisimilarity - and we argue this is due to the following fact: if we take bisimilar transition systems $T$ and $F$ such that $F$ has finitely many states, then for each reachable state $t$ of $T$ there is a bisimilar reachable state $f$ of $F$. In other words, $F$ gives a complete characterization of all reachable states of $T$. This is no more true for the other equivalences of van Glabbeek's hierarchy; if we take e.g., trace equivalence (see Definition 8 ) and two transition systems $T$ and $F$ such that $T$ and 


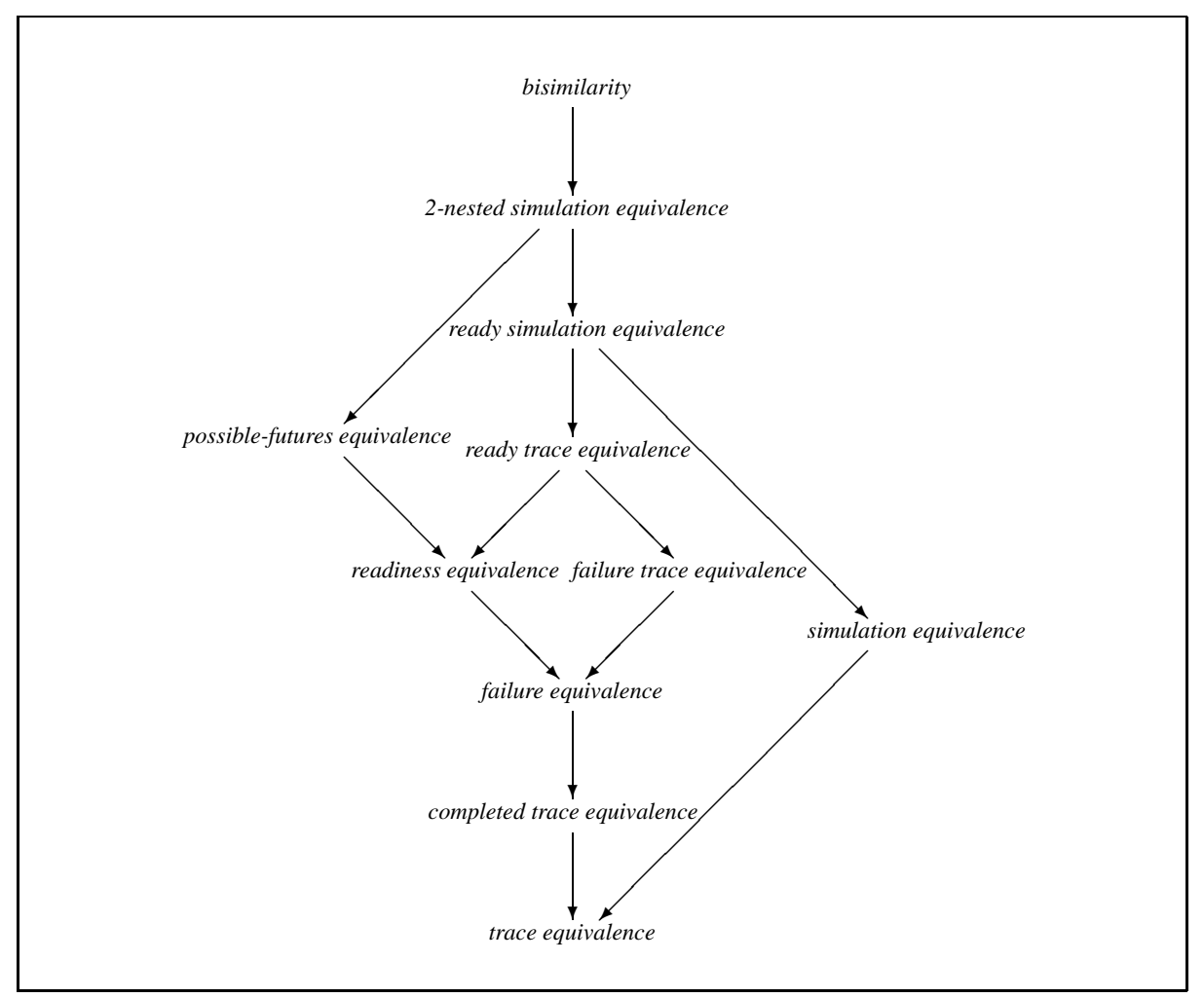

Fig. 1. van Glabbeek's hierarchy of behavioural equivalences 
$F$ are trace equivalent and $F$ has finitely many states, then the only thing we can say about $T$ and $F$ is that their roots have the same sets of traces-but if we take a reachable state $t$ of $T$, it need not be trace equivalent to any reachable state of $F$. If we want to check some temporal property of $T$ (e.g., something bad never happens), then we are usually interested in all reachable states of $T$. It is thus sensible to ask whether there is a finite-state transition system $F^{\prime}$ such that each reachable state of $T$ is equivalent to some state of $F^{\prime}$. If so, we can examine features of $F^{\prime}$ instead of $T$ and as $F^{\prime}$ has only finitely many states, it should be easier. This is the basic idea which leads to the notion of finite characterization.

Definition 3. Let $T$ be a transition system and let $\leftrightarrow$ be an equivalence over $\mathcal{T}$. A finitestate transition system $F$ is a finite characterization of $T$ w.r.t. $\leftrightarrow$ if all the following conditions are true: $T \leftrightarrow F$, states of $F$ are pairwise nonequivalent w.r.t. $\leftrightarrow$, and for each reachable state $t$ of $T$ there is a reachable state $f$ of $F$ such that $t \leftrightarrow f$.

Now we examine the question when finite characterizations exist and what is their relationship with finite representations. First we need to introduce further notions.

Definition 4. Let $\leftrightarrow$ be an equivalence over $\mathcal{T}$. For each transition system $T=(S$, Act $\rightarrow, r)$ we define the transition system $T / \leftrightarrow=\left(S^{\prime}\right.$, Act $\left., \rightarrow^{\prime}, r^{\prime}\right)$ in the following way:

- $S^{\prime}$ contains equivalence classes of $S / \leftrightarrow$ (the equivalence class containing $s \in S$ is denoted by $[s]$ ).

- The relation $\rightarrow^{\prime}$ is determined by the rule $s \stackrel{a}{\rightarrow} t \Longrightarrow[s] \stackrel{a}{\rightarrow}[t]$.

$-r^{\prime}=[r]$

The equivalence $\leftrightarrow$ is said to have quotients if for any $T \in \mathcal{T}$ the natural projection $p: T \longrightarrow T / \leftrightarrow$, assigning to each state $s$ of $T$ the state $[s]$ of $T / \leftrightarrow$, is a part of $\leftrightarrow$ (i.e., $s \leftrightarrow[s]$ for each state $s$ of $T$ ).

The notion of finite characterization is naturally motivated. Now we can ask what features of a transition system $T$ guarantee an existence of a finite characterization of $T$. This is the aim of the following definition:

Definition 5. Let $\leftrightarrow$ be an equivalence over $\mathcal{T}$. A transition system $T$ is strongly regular w.r.t. $\leftrightarrow$ if $T$ can reach only finitely many states up to $\leftrightarrow$.

The next lemma says when the condition of strong regularity guarantees an existence of a finite characterization.

Lemma 1. Let $\leftrightarrow$ be an equivalence over $\mathcal{T}$ which has quotients. Then $T$ has a finite characterization w.r.t. $\leftrightarrow$ iff $T$ is strongly regular w.r.t. $\leftrightarrow$.

Proof.

" $\Rightarrow$ " Obvious.

“ $\Leftarrow$ ” As $T$ is strongly regular w.r.t. $\leftrightarrow$ and $\leftrightarrow$ has quotients, the transition system $T / \leftrightarrow$ is a finite characterization of $T$. 
Now we prove that the requirement of "having quotients" from the previous lemma is not too restrictive in fact—all equivalences of van Glabbeek's hierarchy have this property. Due to the lack of space we cannot give a separate proof for each of them; instead we present just two full proofs which "cover" the whole hierarchy in the sense that all remaining proofs can be obtained by slight modifications of one of the two indicated approaches.

Definition 6. Let $T=(S, A c t, \rightarrow, r)$ be a transition system. For each state $s \in S$ we define the set $I(s)=\{a \in$ Act $\mid \exists t \in S$ such that $s \stackrel{a}{\rightarrow} t\}$. A pair $(w, \Phi) \in$ Act ${ }^{*} \times \mathcal{P}($ Act $)$ is a failure pair of $T$, if there is a state $s \in S$ such that $r \stackrel{w}{\rightarrow} s$ and $I(s) \cap \Phi=\emptyset$. Let $F(T)$ denote the set of all failure pairs of $T$. Transition systems $T_{1}, T_{2}$ are failure equivalent, written $T_{1}={ }_{f} T_{2}$, if $F\left(T_{1}\right)=F\left(T_{2}\right)$

Lemma 2. Failure equivalence has quotients.

Proof. Let $T=(S, A c t, \rightarrow, r)$ be a transition system. We show that $F(p)=F([p])$ for each state $p \in S$.

"ᄃ": Let $(w, \Phi) \in A c t^{*} \times \mathcal{P}(A c t)$ be a failure pair of $p$. By definition, there is a state $p^{\prime} \in S$ such that $p \stackrel{w}{\rightarrow} p^{\prime}$ and $I\left(p^{\prime}\right) \cap \Phi=\emptyset$. But then also $[p] \stackrel{w}{\rightarrow}\left[p^{\prime}\right]$. The set $I\left(\left[p^{\prime}\right]\right)$ is the union of all $I(q)$ such that $q \in\left[p^{\prime}\right]$. As $u={ }_{f} v$ implies $I(u)=I(v)$, we can conclude that $I\left(\left[p^{\prime}\right]\right)=I\left(p^{\prime}\right)$, hence $I\left(\left[p^{\prime}\right]\right) \cap \Phi=\emptyset$, thus $(w, \Phi) \in F([p])$.

" $\supseteq$ ": Let $(w, \Phi) \in A c t^{*} \times \mathcal{P}(A c t)$ be a failure pair of $[p]$ and let $w=a_{k} \ldots a_{1}$. By definition, there is a sequence of transitions $\left[p_{k}\right] \stackrel{a_{k}}{\rightarrow}\left[p_{k-1}\right] \stackrel{a_{k-1}}{\rightarrow} \ldots \stackrel{a_{1}}{\rightarrow}\left[p_{0}\right]$ in $T /=_{f}$ such that $p \in\left[p_{k}\right]$ and $I\left(\left[p_{0}\right]\right) \cap \Phi=\emptyset$. We show that for each state $q$ of $T$ such that $q \in\left[p_{i}\right]$, where $i \in\{0, \ldots, k\}$, the pair $\left(a_{i} \ldots a_{1}, \Phi\right)$ belongs to $F(q)$. We proceed by induction on $i$ :

- $\mathbf{i}=\mathbf{0}:$ as $I(q)=I\left(\left[p_{0}\right]\right)$, we have $(\epsilon, \Phi) \in F(q)$.

- induction step: as $\left[p_{i}\right] \stackrel{a_{i}}{\rightarrow}\left[p_{i-1}\right]$, there are states $u, v$ of $T$ such that $u \stackrel{a_{i}}{\rightarrow} v$, $u \in\left[p_{i}\right]$ and $v \in\left[p_{i-1}\right]$. By induction hypothesis we have $\left(a_{i-1} \ldots a_{1}, \Phi\right) \in F(v)$, hence $\left(a_{i} \ldots a_{1}, \Phi\right) \in F(u)$. As $q={ }_{f} u$, the pair $\left(a_{i} \ldots a_{1}, \Phi\right)$ belongs to $F(q)$.

The same technique can be also applied to trace equivalence, completed trace equivalence, readiness equivalence, failure trace equivalence, ready trace equivalence and possible-futures equivalence.

Definition 7. Let $T_{1}=\left(S_{1}\right.$, Act $\left._{1}, \rightarrow_{1}, r_{1}\right)$ and $T_{2}=\left(S_{2}\right.$, Act $\left._{2}, \rightarrow_{2}, r_{2}\right)$ be transition systems. A relation $R \subseteq S_{1} \times S_{2}$ is a simulation if whenever $(s, t) \in R$ then

$$
\forall a \in A t_{1}: s \stackrel{a}{\rightarrow}_{1} s^{\prime} \Longrightarrow \exists t^{\prime}: t \stackrel{a}{\rightarrow}_{2} t^{\prime} \wedge\left(s^{\prime}, t^{\prime}\right) \in R
$$

Transition system $T_{1}$ is simulated by $T_{2}$, written $T_{1} \sqsubseteq_{s} T_{2}$, if there is a simulation $R$ with $\left(r_{1}, r_{2}\right) \in R$. It is easy to see that $\bigsqcup_{s}$ is a preorder. Transition systems $T_{1}, T_{2}$ are simulation equivalent, written $T_{1}={ }_{s} T_{2}$, if $T_{1} \sqsubseteq_{s} T_{2}$ and $T_{2} \sqsubseteq_{s} T_{1}$.

Lemma 3. Simulation equivalence has quotients. 
Proof. Let $T=(S, A c t, \rightarrow, r)$ be a transition system. We show that $t={ }_{s}[t]$ for each state $t \in S$. By definition, we must show an existence of two simulations $P, R$ such that $(t,[t]) \in P$ and $([t], t) \in R$. The simulation $P$ is exactly the natural projection $p: T \rightarrow T /=_{s}$, i.e., $P=\{(u,[u]): u \in S\}$. It is easy to check that $P$ is a simulation. The simulation $R$ is defined as follows:

$$
([u], v) \in R \stackrel{\text { def }}{\Longleftrightarrow} \exists p \in[u]: p \sqsubseteq_{s} v
$$

We prove that $R$ is indeed a simulation. Suppose $[u] \stackrel{a}{\rightarrow}\left[u^{\prime}\right]$. By definition of $T /=_{s}$, there are $q, q^{\prime} \in S$ such that $q \stackrel{a}{\rightarrow} q^{\prime}, u={ }_{s} q$, and $u^{\prime}={ }_{s} q^{\prime}$. Moreover, by definition of $R$ there is $p \in S$ with $p={ }_{s} q$ and $p \sqsubseteq_{s} v$. As $q \sqsubseteq_{s} p \sqsubseteq_{s} v$, we also have $q \sqsubseteq_{s} v$ by transitivity of $\sqsubseteq_{s}$. Hence $v \stackrel{a}{\rightarrow} v^{\prime}$ for some $v^{\prime} \in S$ with $q^{\prime} \sqsubseteq_{s} v^{\prime}$. As $q^{\prime} \in\left[u^{\prime}\right]$, the pair ( $\left.\left[u^{\prime}\right], v^{\prime}\right)$ belongs to $R$ and the proof is finished.

This method also works for ready simulation equivalence and 2-nested simulation equivalence. As bisimilarity has quotients (this is obvious), we can now state the following theorem:

Theorem 1. Each equivalence in van Glabbeek's hierarchy has quotients.

There are also other well-known equivalences which have quotients, e.g., weak bisimilarity (see [Mil89]) or branching bisimilarity (see [vGW89]). But this property is naturally not general - there are also equivalences which do not have quotients. To present a concrete example, we first need several definitions.

Definition 8. Let $T=(S$, Act $, \rightarrow, r)$ be transition system. A trace of $T$ is any sequence $w \in \Sigma^{+}$such that $r \stackrel{w}{\rightarrow}$ s for some $s \in S$. A trace $w$ of $T$ is completed if $r \stackrel{w}{\rightarrow} s$ for some $s \in S$ which does not have any successors. Transition systems $T_{1}, T_{2}$ are

- trace equivalent if they have the same sets of traces.

- completed trace equivalent if they have the same sets of traces and the same sets of completed traces.

- language equivalent, written $T_{1}={ }_{L} T_{2}$, if they have the same sets of completed traces.

Language equivalence is well-known from the theory of formal languages and automata. Note that it is incomparable even with trace equivalence.

Theorem 2. Language equivalence does not have quotients.

Proof. A simple counterexample looks as follows:
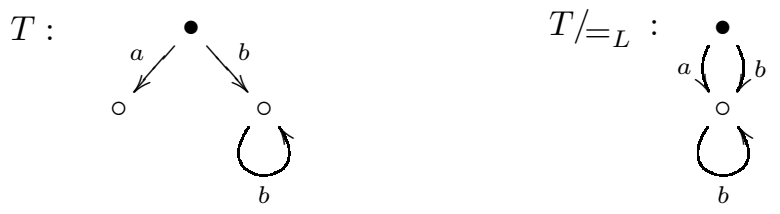

Clearly $r \neq_{L}[r]$ because the set of completed traces of $r$ is $\{a\}$ while the set of completed traces of $[r]$ is empty. 
We have seen that if we restrict our attention to behavioural equivalences which have quotients, then the condition of strong regularity becomes necessary and sufficient for an existence of a finite characterization. An interesting question is, what is the exact relationship between conditions of regularity and strong regularity. First, we already know that there are equivalences for which these two conditions coincide (e.g., bisimilarity, weak bisimilarity or branching bisimilarity). But there are also equivalences for which conditions of regularity and strong regularity express different properties.

Theorem 3. Let $\leftrightarrow$ be an equivalence of van Glabbeek's hierarchy which lies under bisimilarity. Then there is a transition system $T$ such that $T$ is regular w.r.t. $\leftrightarrow$ and $T$ is not strongly regular w.r.t. $\leftrightarrow$.

Proof. (sketch) Transition systems $T_{3}$ and $T_{4}$ of Figure 2 are ready simulation equivalent. As $T_{4}$ has finitely many states, $T_{3}$ is regular w.r.t. all equivalences which lie under ready simulation equivalence in van Glabbeek's hierarchy. At the same time we may observe that $T_{3}$ can reach infinitely many states which are pairwise nonequivalent w.r.t. trace equivalence. Hence $T_{3}$ is not strongly regular w.r.t. any equivalence in van Glabbeek's hierarchy.

Similarly, $T_{1}$ and $T_{2}$ are 2-nested simulation equivalent, but $T_{1}$ can reach infinitely many states which are pairwise nonequivalent w.r.t. possible-futures equivalence. Hence $T_{1}$ is regular w.r.t. possible-futures equivalence and 2-nested simulation equivalence, but not strongly regular w.r.t. the mentioned equivalences.

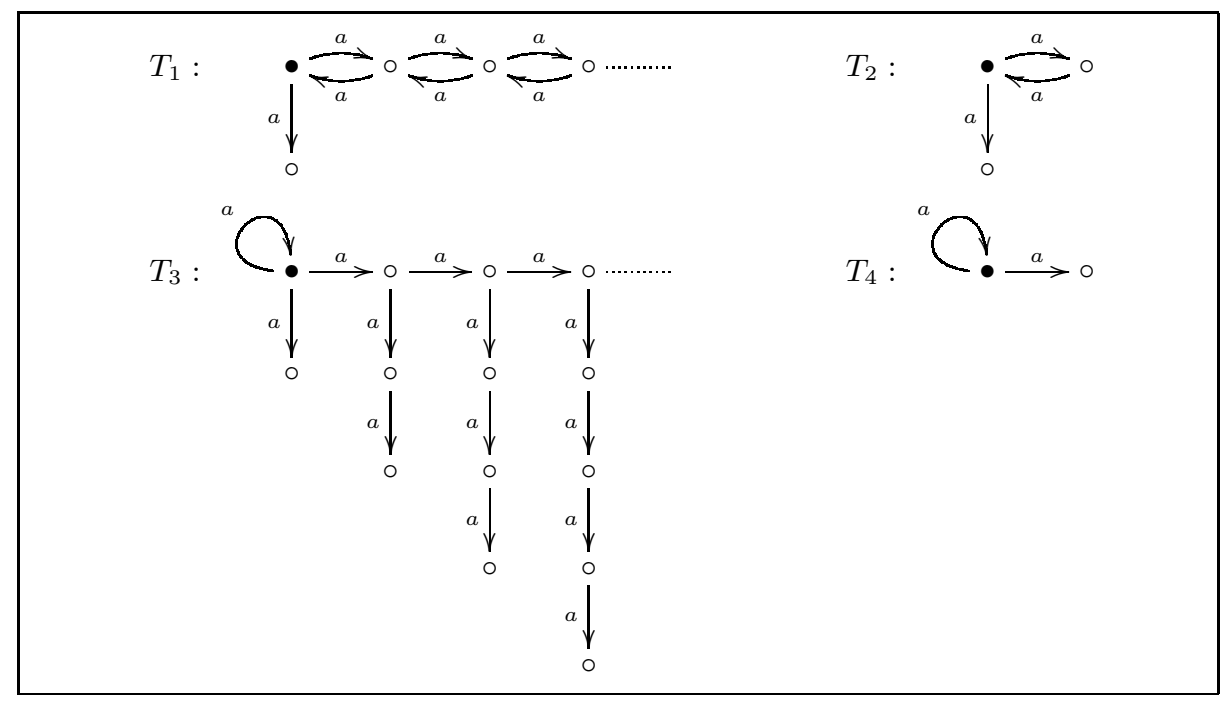

Fig. 2. Transition systems from the proof of Theorem 3 


\section{Future work}

An open problem is whether the notions of regularity and strong regularity have different decidability features. However, this area seems to be quite unexplored. The notions of finite characterization and strong regularity surely deserve a deeper examination, and this is the subject we would like to work on in the future.

\section{Acknowledgement}

Most of the presented work has originated in discussions with Mogens Nielsen during my stay at Aarhus University (BRICS). I am very grateful for his kind supervision and advice. Thanks are also due to anonymous referees for constructive comments.

\section{References}

[BCS96] O. Burkart, D. Caucal, and B. Steffen. Bisimulation collapse and the process taxonomy. In Proceedings of CONCUR'96, volume 1119 of LNCS, pages 247-262. SpringerVerlag, 1996.

[BG96] D.J.B. Bosscher and W.O.D. Griffionen. Regularity for a large class of context-free processes is decidable. In Proceedings of ICALP'96 [Ica96], pages 182-192.

[ČKK97] I. Černá, M. Křetínský, and A. Kučera. Bisimilarity is decidable in the union of normed BPA and normed BPP processes. Electronic Notes in Theoretical Computer Science, 6, 1997.

[Ica96] Proceedings of ICALP'96, volume 1099 of LNCS. Springer-Verlag, 1996.

[Jan97] P. Jančar. Bisimulation equivalence is decidable for one-counter processes. In Proceedings of ICALP'97, volume 1256 of LNCS, pages 549-559. Springer-Verlag, 1997.

[JE96] P. Jančar and J. Esparza. Deciding finiteness of Petri nets up to bisimilarity. In Proceedings of ICALP'96 [Ica96], pages 478-489.

[Kuč96] A. Kučera. Regularity is decidable for normed PA processes in polynomial time. In Proceedings of FST\&TCS'96, volume 1180 of LNCS, pages 111-122. Springer-Verlag, 1996.

[Kuč97] A. Kučera. How to parallelize sequential processes. In Proceedings of CONCUR'97, volume 1243 of $L N C S$, pages 302-316. Springer-Verlag, 1997.

[Mil89] R. Milner. Communication and Concurrency. Prentice-Hall, 1989.

[MM94] S. Mauw and H. Mulder. Regularity of BPA-systems is decidable. In Proceedings of CONCUR'94, volume 836 of LNCS, pages 34-47. Springer-Verlag, 1994.

[vG90] R.J. van Glabbeek. The linear time-branching time spectrum. In Proceedings of CONCUR'90, volume 458 of LNCS, pages 278-297. Springer-Verlag, 1990.

[vGW89] R.J. van Glabbeek and W.P. Weijland. Branching time and abstraction in bisimulation semantics. Information Processing 89, pages 613-618, 1989. 